\title{
MicroRNA dysregulation in the tumor microenvironment influences the phenotype of pancreatic cancer
}

\author{
Eva Karamitopoulou ${ }^{1}$, Stefan Haemmig ${ }^{2}$, Ulrich Baumgartner ${ }^{2}$, Cornelia Schlup ${ }^{2}$, \\ Martin Wartenberg ${ }^{1}$ and Erik Vassella ${ }^{2}$ \\ ${ }^{1}$ Division of Clinical Pathology, Institute of Pathology, University of Bern, Bern, Switzerland and ${ }^{2}$ Molecular \\ Pathology Unit, Institute of Pathology, University of Bern, Bern, Switzerland
}

\begin{abstract}
Cellular interactions in the tumor microenvironment influence neoplastic progression in pancreatic ductal adenocarcinoma. One underlying mechanism is the induction of the prognostically unfavorable epithelialmesenchymal-transition-like tumor budding. Our aim is to explore the expression of microRNAs implicated in the regulation of tumor budding focusing on the microenvironment of the invasive front. To this end, RNA from lasercapture-microdissected material of the main tumor, tumor buds, juxta-tumoral stroma, tumor-remote stroma, and non-neoplastic pancreatic parenchyma from pancreatic cancer cases with $(n=7)$ and without $(n=6)$ tumor budding was analyzed by qRT-PCR for the expression of a panel of miRNAs that are known to be implicated in the regulation of epithelial-mesenchymal transition, including miR-21, miR-183, miR-200b, miR-200c, miR-203, miR-205, miR-210, and miR-217. Here we show that at the invasive front of pancreatic ductal adenocarcinoma, specific microRNAs, are differentially expressed between tumor buds and main tumor cells and between cases with and without tumor budding, indicating their involvement in the regulation of the budding phenotype. Notably, miR-200b and miR-200c were significantly downregulated in the tumor buds. Consistent with this finding, they negatively correlated with the expression of epithelial-mesenchymal-transition-associated E-cadherin repressors ZEB1 and ZEB2 in the budding cells $(P<0.001)$. Interestingly, many microRNAs were also dysregulated in juxta-tumoral compared to tumor-remote stroma suggesting that juxta-tumoral stroma contributes to microRNA dysregulation. Notably, miR-200b and miR-200c were strongly downregulated while miR-210 and miR-21 were upregulated in the juxtatumoral vs tumor-remote stroma in carcinomas with tumor budding. In conclusion, microRNA targeting in both tumor and stromal cells could represent a treatment option for aggressive pancreatic cancer.
\end{abstract}

Modern Pathology (2017) 30, 1116-1125; doi:10.1038/modpathol.2017.35; published online 26 May 2017

Our understanding of the contribution of the tumor microenvironment to cancer in general and pancreatic ductal adenocarcinoma in particular has been broadened based on recent data. ${ }^{1-6}$ Many of the cellular interactions within the tumor microenvironment support enhanced growth and dissemination of cancer cells. ${ }^{2-6}$ Especially, the emerging role of stromal cells in the establishment of a tumorpermissive environment has opened a great potential for therapeutic intervention. In pancreatic ductal adenocarcinoma, which is characterized by dismal

Correspondence: Dr E Karamitopoulou, MD, Division of Clinical Pathology, Institute of Pathology, University of Bern, Murtenstrasse 31, CH-3008 Bern, Switzerland.

E-mail: eva.diamantis@pathology.unibe.ch

Received 27 December 2016; revised 20 March 2017; accepted 20 March 2017; published online 26 May 2017 prognosis and a rich stromal component, targeting of the tumor microenvironment is increasingly becoming an attractive therapeutic option, because of lack of efficacy of standard chemotherapy.

Especially at the invasive front of aggressive gastrointestinal cancers, including pancreatic cancer, tumor cells are represented by a population of dedifferentiated cancer cells with epithelial-mesenchymal-transition-like features and dissociative growth, coined tumor budding cells. ${ }^{7-10}$ Epithelial-mesenchymaltransition-like tumor budding is a strong and independent prognostic factor in pancreatic cancer. ${ }^{9,10}$ The microenvironment surrounding tumor buds is therefore especially interesting, since it probably has a distinguished role in supporting tumor budding cells, promoting their migration, angioinvasion, and metastatic potential. However, the molecular mechanism underlying tumor budding remains currently unclear. 
MicroRNAs are a class of non-coding RNAs involved in key cellular processes such as proliferation, apoptosis, and differentiation. ${ }^{11,12}$ These small ( 18-25 nucleotides) RNAs may bind to target mRNAs by simple Watson-Crick base-pairing to repress their proper posttranscriptional maturation process. It is thought that individual microRNAs can target multiple genes implicated in diverse cellular functions, suggesting that microRNAs play important roles in a wide variety of cellular processes. Because of their role in gene regulation at the posttranscriptional level, microRNAs are potential clinical biomarkers for human cancers as well as targets for molecular therapy. ${ }^{13-15}$ Restoration or repression of microRNA expression and activity is very promising in managing cancer, and many studies on pre-clinical models have demonstrated the feasibility and efficacy of microRNA-based therapy..$^{15-17}$

Here we aim at investigating the expression profile of specific microRNAs originating from different cell populations within the tumor microenvironment of the invasive front of pancreatic ductal adenocarcinoma with special focus on epithelial-mesenchymaltransition-associated microRNAs. Therefore, the expression of these microRNAs was analyzed in cases with and without tumor budding in microdissected material from the main tumor mass, the tumor budding cells (in cases with budding), the juxtatumoral and tumor-remote stroma, and the matched normal pancreatic parenchyme. We show that some of these microRNAs are differentially regulated between normal tissue and tumor, between main tumor cells and budding cells, as well as between juxta-tumoral and tumor-remote stroma, suggesting a crosstalk between tumor cells and their surrounding stromal environment through microRNAs.

\section{Material and methods}

\section{Patients and Tissues}

Histomorphological data from 120 non-pretreated pancreatic ductal adenocarcinomas, surgically resected between 2002 and 2010, were reviewed from the corresponding hematoxylin and eosin stained slides, while clinical data were obtained from corresponding reports. Clinicopathological information included age, gender, tumor diameter, number of positive lymph nodes, and total number of lymph nodes collected, TNM stage (8th Edition, 2017), ${ }^{18}$ perineural, blood vessel and lymphatic invasion, and resection margin status (R-status) (Supplementary Table 1). The use of the material was approved by the local Ethics committee of the University of Bern.

\section{Construction of Tissue Microarrays}

Tissue microarrays were built as previously described. ${ }^{19}$ To reduce bias due to heterogeneity, at least four tumor punches from the tumor center and the invasive tumor front of each patient was included on this array.

\section{Immunohistochemistry}

Tissue microarray blocks were cut at $4 \mu \mathrm{m}$ and sections were de-waxed and re-hydrated in $\mathrm{dH}_{2} \mathrm{O}$. Sections were stained immunohistochemically for E-cadherin (1:200; Dako, Glostrup, Denmark), ZEB1 (1:200; Sigma-Aldrich, St Louis, CA, USA), and ZEB2 (1:400; Sigma-Aldrich). Antigen retrieval was performed with Tris-HCl, $\mathrm{pH} 9$ for $30 \mathrm{~min}$ at $95^{\circ} \mathrm{C}$. Antibody testing and staining protocols have been established and staining was performed by an automated Leica Bond RX System with the Bond Polymer Refine Kit (with DAB as chromogen) and Bond Polymer Refine Red Detection Kit for the double staining (Leica Biosystems, Newcastle, UK). Immunohistochemistry was evaluated by estimating the percentage of positive cells per punch. In the case of multiple tumor punches per localization, the average protein expression was calculated across all punches from the same localization. Evaluation was performed blinded to clinical end points.

\section{Assessment of Tumor Budding}

Tumor budding, defined as detached single cells or clusters of less than five cells, has been evaluated in the course of another project. ${ }^{10}$ Briefly, whole-tissue sections of each case underwent immunohistochemistry for pancytokeratin staining (1:100, clone AE1/ AE3, Monosan, Uden, the Netherlands) and were evaluated for tumor budding using a 10-in-10 approach: The 10 densest hotspots of tumor budding were identified and buds were counted at high magnification $\left(\times 40,0.55 \mathrm{~mm}^{2}\right)$. The average number of buds per case was obtained. Using a receiver operating characteristic curve approach, a cutoff score of 10 buds on average was identified as most discriminatory for survival. Cases with an average of $>10$ buds were classified as 'high' budders; those with $\leq 10$ buds were defined as 'low' budders (Supplementary Figure 1). ${ }^{9}$

\section{MicroRNAs Expression Analysis}

Tumor specimens (seven cases with and six cases without tumor budding) underwent laser capture microdissection to obtain tissue from the main tumor mass, the tumor buds as well as the juxta-tumoral and the tumor-remote stroma (at least $0.5 \mathrm{~cm}$ away from the tumor). The cases were randomly selected among our high-grade budding and non-budding tumor specimens. To better visualize tumor buds, a monoclonal antibody directed against pancytokeratin (1:100, clone MNF116, Dako, Glostrup, Denmark) was used. All 13 cases underwent immunohistochemistry using a high salt protocol, which protects 


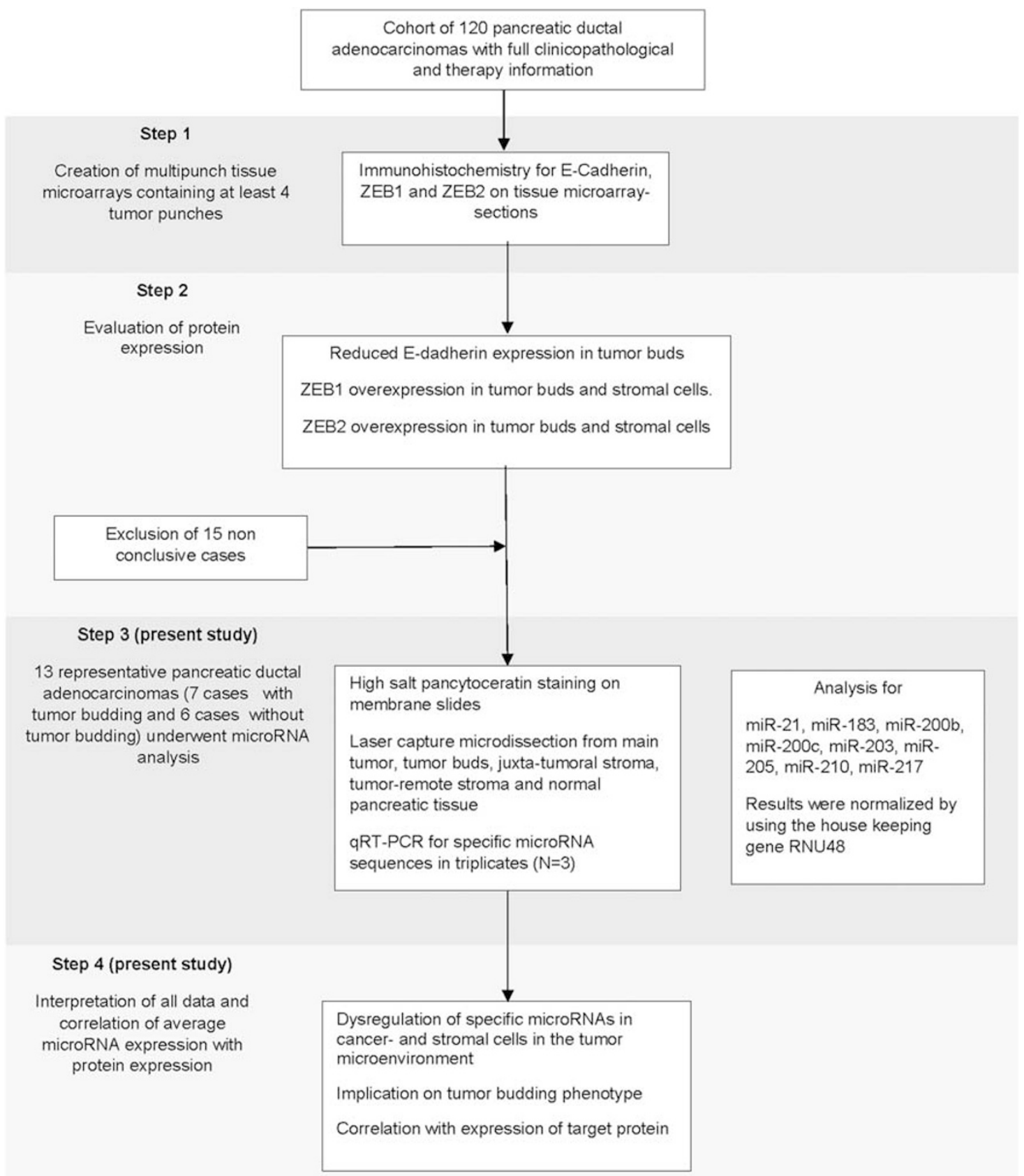

Figure 1 Study design.

microRNAs from degradation by RNases. ${ }^{19}$ RNA was extracted and subjected to a demodification protocol using RecoverAll Total Nucleic Acid Isolation kit (AM1975, Life Technologies) as described. ${ }^{20} \mathrm{~A}$ measure of $120 \mathrm{ng}$ t-RNA was added as carrier RNA

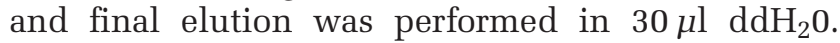
Accurate microRNA expression analysis was performed by pre-amplification following the manufacturer's recommendation, 1:3 dilution of the preamplified product, followed by qRT-PCR using TaqMan technology (Life Technologies). We confirmed that pre-amplification has no significant influence on the relative expression of the analyzed micrRNAs (data not shown). Results are shown relative to the expression of the housekeeping gene RNU48 $(N=3)$.

Analyses were carried out using SAS V9.2 (The SAS Institue, NC, Cary). $P$-values $<0.05$ were considered statistically significant.

The study design is shown in Figure 1. The study was designed to comply with the REMARK guidelines for tumor marker prognostic studies. ${ }^{21}$ 


\section{Results}

\section{Epithelial-Mesenchymal-Transition-Associated Markers}

Expression of the epithelial-mesenchymal-transition-associated markers E-cadherin, ZEB1 and ZEB2, in the tumor buds has been analyzed for the needs of a previous study. ${ }^{19}$ Briefly, pancreatic cancer cases with absent or low density of tumor budding showed preservation of the membranous E-cadherin and markedly reduced nuclear ZEB1 and ZEB2. On the contrary, cases with high density of tumor buds at the invasive front showed a reduced membranous expression of E-cadherin with almost complete loss of protein expression in the tumor buds (Supplementary Figure 1). In addition, an increased nuclear ZEB1 and ZEB2 expression was observed, especially in the budding cells and the stromal cells surrounding them.

\section{Expression Analysis of MicroRNAs in the Main Tumor and Tumor Buds}

The expression of a panel of microRNAs that are known to be implicated in the regulation of epithelial-mesenchymal transition in pancreatic cancer, including miR-21, miR-183, miR-200b, miR-200c, miR-203, miR-205, miR-210, and miR-217, was analyzed by qRT-PCR from RNA of laser-capturemicrodissected material including the main tumor, tumor buds, and matched normal tissue (Figure 2).

Mir-200 family members miR-200b, miR-200c, and miR-205 are known regulators of E-cadherin repressors ZEB1 and ZEB2. ${ }^{22-27}$ In cases with tumor budding, miR-200c and miR-205 were significantly downregulated both in the main tumor and the tumor buds compared to normal tissue $(P<0.001)$, while miR-200b was downregulated in the tumor buds compared to the main tumor $(P<0.05)$. On the contrary, in cases without budding both miR-200c and miR-200b were overexpressed in the tumor cells, while no dysregulation of miR-205 was observed.

Interestingly, qRT-PCR analysis revealed that miR-203 and miR-200b gave rise to a similar expression profile and were downregulated in the tumor buds compared to the main tumor of cases with budding, without being downregulated in the main tumor itself. miR-183, another microRNA that is able to target ZEB1 (ref. 28), was upregulated in all tumors independently of tumor buddng.

In addition, miR-21 and miR-210 were overexpressed in the tumor cells (including tumor budding cells), compared to normal $(P<0.001)$ in all cases independently of tumor budding. However, miR-21 showed an aggravated upregulation in the tumor buds compared to main tumor in cases with budding. MiR-217 was significantly downregulated in the tumor cells compared to normal, in all cases $(P<0.0001)$ independently of tumor budding. The

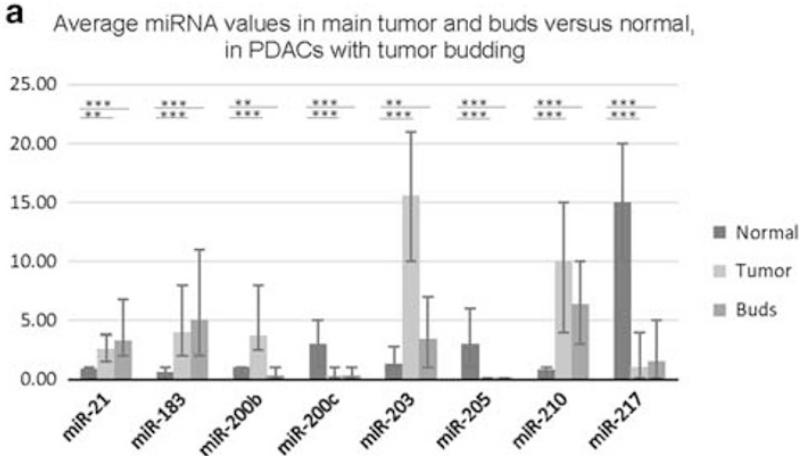

b Average miRNA values in tumor versus normal, in PDACs without tumor budding

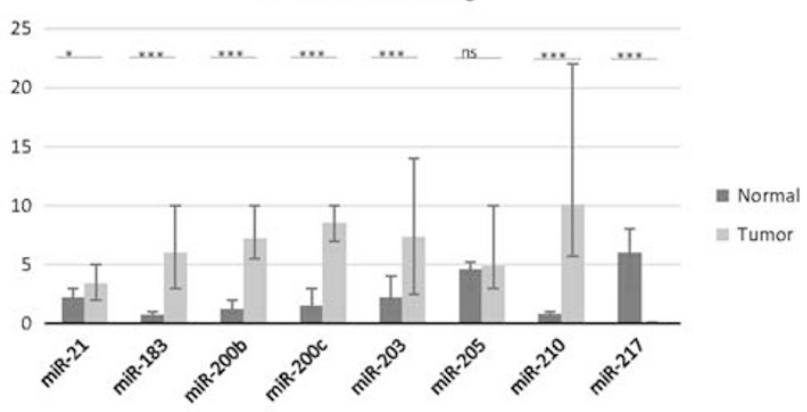

ns: $p>0.05 ;{ }^{\star}: p<0.05 ; * \star p<0.01 ; * \star *: p<0.001$

Figure 2 Expression of miRNAs in the tumor cells, in pancreatic ductal adenocarcinomas with (a) and without (b) tumor budding, in comparison with non-neoplastic pancreatic parenchyma (normal).

average expression level and range of the examined microRNAs in the several cell populations are depicted in Table 1 (cases with tumor budding) and Table 2 (cases without tumor budding).

\section{Correlation of Expression of ZEB1 and ZEB2 with Analyzed MiRNAs}

To analyze if microRNAs are able to induce epithelial-mesenchymal transition by targeting $Z E B 1$ and/or ZEB2 genes, we assessed whether the expression of these microRNAs are correlated inversely with the expression of ZEB1 and ZEB2 proteins. To this end, serial tissue microarray sections were analyzed by IHC for ZEB1 and ZEB2 proteins and results were compared to the microRNA expression. According to our findings, ${ }^{19}$ the expression of both proteins was induced in the tumor buds in cases with tumor budding. A negative correlation was found between miR-200c, miR-200b, and miR-205 levels and ZEB1 and ZEB2 expression by the tumor budding cells $(P<0.001$ and $P<0.005$, respectively) (Figure 3 and Supplementary Figure 2), suggesting that ZEB1 and ZEB2 are potential targets of these microRNAs, but further studies are necessary to clarify this hypothesis. 
Table 1 Pancreatic ductal adenocarcinomas with epithelial-mesenchymal-transition-like tumor budding

\begin{tabular}{|c|c|c|c|c|c|c|c|c|}
\hline Tissue & $\operatorname{MiR}-21$ & MiR-183 & MiR-200b & $M i R-200 c$ & MiR-203 & MiR-205 & MiR-210 & MiR-217 \\
\hline Normal & $0.9(0.5-1)$ & $0.6(0.1-1)$ & $1(0.1-1)$ & $3(1-5)$ & $1.3(1-2.5)$ & $3(1-6)$ & $0.8(0.1-1)$ & $15(10-20)$ \\
\hline Tumor & $2.6(1.5-3.8)$ & $4(2-8)$ & $3.7(1.5-8)$ & $0.3(0.1-1)$ & $15.6(10-22)$ & $0.1(0.1-0.1)$ & $10(4-15)$ & $1(0.1-4)$ \\
\hline Tumor buds & $3.3(2-6.8)$ & $5(2-11)$ & $0.3(0.1-1)$ & $0.3(0.1-1)$ & $3.4(1-7)$ & $0.1(0.1-0.1)$ & $6.4(3-10)$ & $1.5(0.1-5)$ \\
\hline Juxta-tumoral stroma & $2.5(1.2-5)$ & $1.4(0.8-4)$ & $0.2(0.1-0.3)$ & $0.4(0.2-1)$ & $2.3(1-8)$ & $4(2-10)$ & $5.5(1.8-10)$ & $11.8(5.5-20)$ \\
\hline Tumor-remote stroma & $0.9(0.5-1)$ & $0.5(0.1-1)$ & $0.75(0.1-1)$ & $3.9(1.5-10)$ & $0.5(0.2-2)$ & $4.3(2-10)$ & $0.2(0.1-1)$ & $15(10-20)$ \\
\hline
\end{tabular}

Average values and range of examined microRNAs in the different tissue areas.

Table 2 Pancreatic ductal adenocarcinomas without epithelial-mesenchymal-transition-like tumor budding

\begin{tabular}{|c|c|c|c|c|c|c|c|c|}
\hline Tissue & $M i R-21$ & MiR-183 & $M i R-200 b$ & $M i R-200 c$ & MiR-203 & MiR-205 & $M i R-210$ & $M i R-217$ \\
\hline Normal & $2.2(1-3)$ & $0.7(0.1-1)$ & $1.2(1-2)$ & $1.5(1-3)$ & $2.2(1-5)$ & $4.6(1-8)$ & $0.8(0.1-1)$ & $6(3-8)$ \\
\hline Tumor & $3.4(2-5)$ & $6(3-10)$ & $7.2(5.5-10)$ & $8.5(7-10)$ & $7.3(2.5-14)$ & $4.9(3-10)$ & $10.1(5.5-22)$ & $0.1(0.1-0.1)$ \\
\hline Juxta-tumoral stroma & $4(2-7)$ & $1.6(1-4)$ & $4.2(1-9)$ & $3(1-5)$ & $2(1-4.5)$ & $5.3(2.5-20)$ & $1.5(1-2)$ & $3(1-4)$ \\
\hline Tumor-remote stroma & $2.4(1-4)$ & $0.8(0.2-2)$ & $0.8(0.1-1)$ & $1.5(1-3)$ & $1.2(1-1.5)$ & $4.3(1.5-10)$ & $1.2(0.2-1.5)$ & $6(2-10)$ \\
\hline
\end{tabular}

Average values and range of examined microRNAs in the different tissue areas.
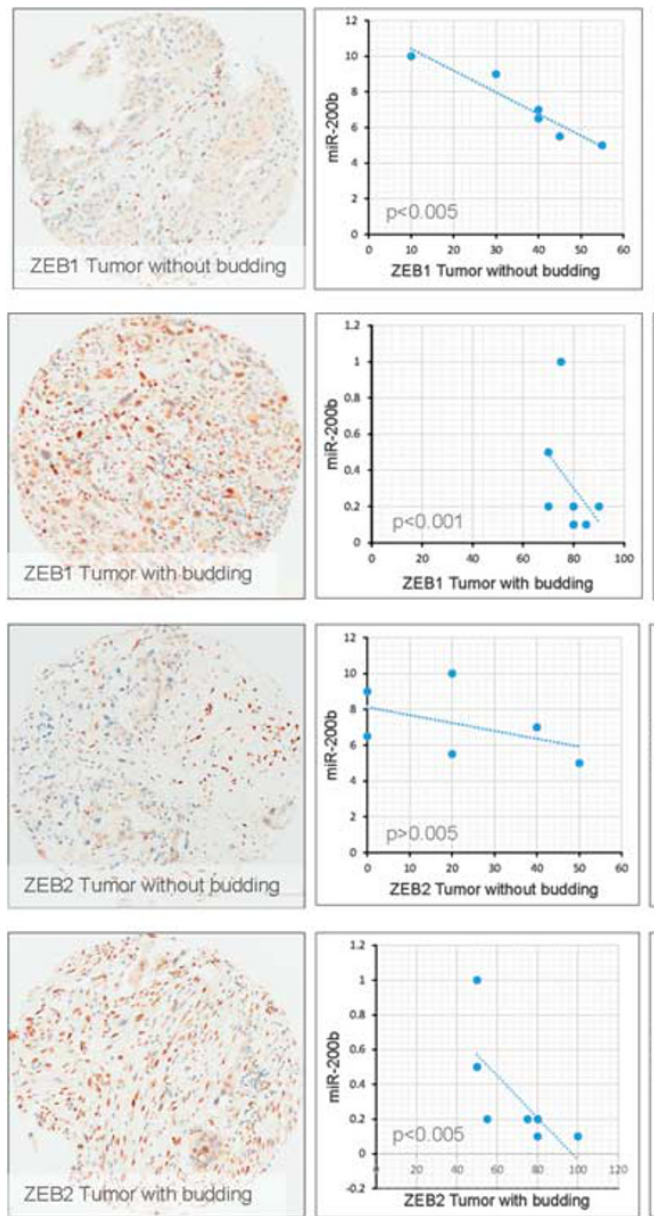
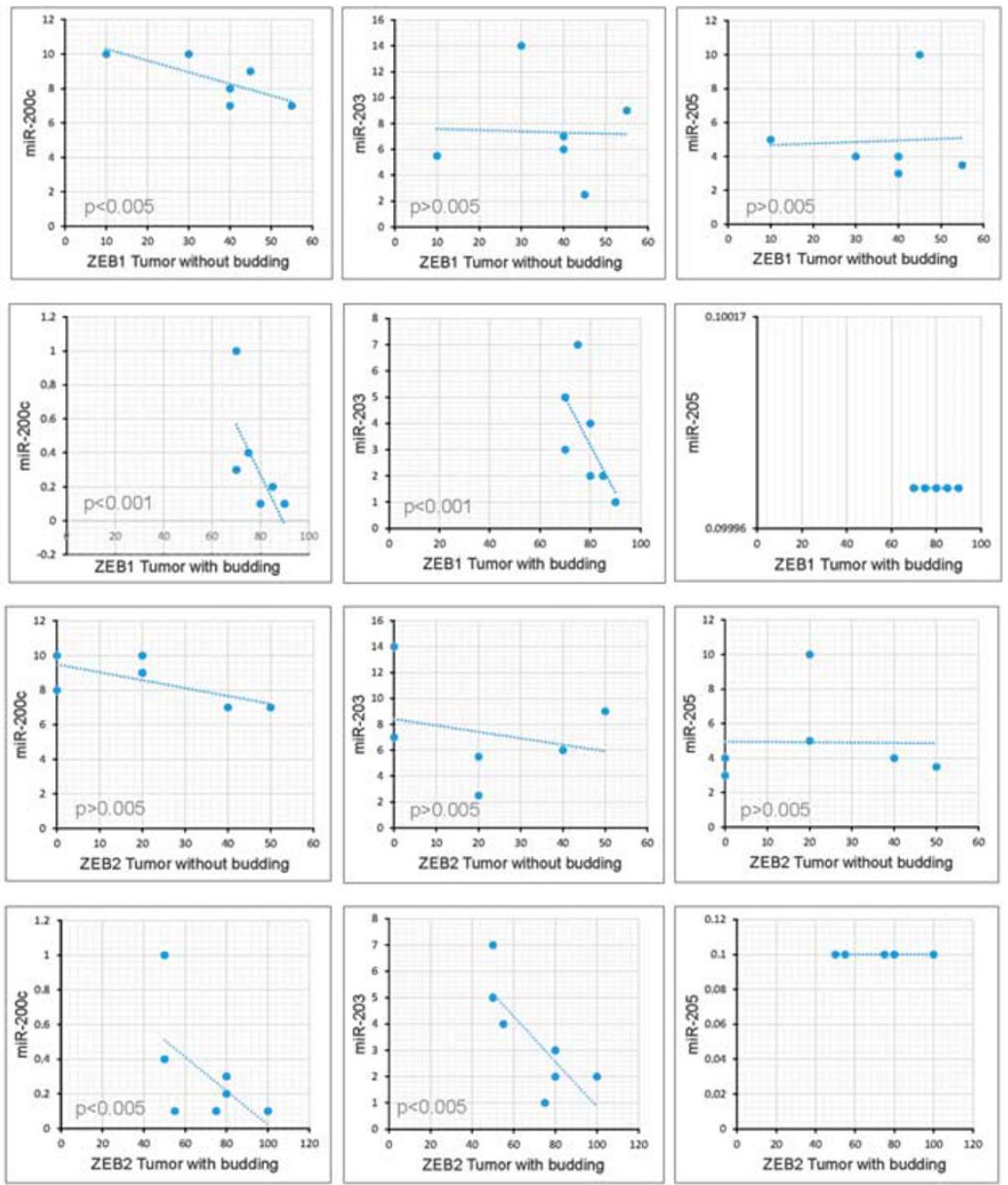

Figure 3 Correlation between ZEB1 and microRNA expression in the tumor cells of cases without (first row) and with tumor budding (second row), as well as between ZEB2 and microRNA expression in the tumor cells of cases without (third row) and with tumor budding (fourth row), concerning miR-200b, miR-200c, miR-203, and miR-205.

Dysregulation of MicroRNAs in the Stromal Cells. Juxta-tumoral and tumor-remote stromal cells were also compared for microRNA expression. Interestingly, many of the microRNAs were differentially regulated in the juxta-tumoral stromal cells compared to the tumor-remote stroma. MiR-200c and miR-200b were downregulated in the juxta-tumoral stroma compared to tumor-remote stroma $(P<0.001)$ 
in cases with tumor budding and upregulated in cases without tumor budding compared to tumorremote stroma (Figure 4).

MiR-210 showed increased expression in juxtatumoral stromal cells compared to tumor-remote stroma only in cases with tumor budding, while miR-21, miR-183, and miR-203 were found to be upregulated in the juxta-tumoral stroma in all cases, independently of the presence of tumor budding. MiR-217 was underexpressed in juxta-tumoral stroma in all cases independently of tumor budding, while miR-205 was not found to be dysregulated in the stromal cells in any of our cases. No microRNA dysregulation was found in the tumor-remote stroma (Figure 4).

In conclusion, our results suggest that tumor and stromal cells communicate via microRNAs. To assess if this communication network affects the expression of target genes, ZEB1 and ZEB2 were analyzed in juxta-tumoral and tumor-remote stromal cells. A negative correlation was found between miR-200b and miR-200c expression and ZEB1 and ZEB2 expression in the juxta-tumoral stromal cells $(P<0.005)$ (Figure 5 and Supplementary Figure 3).

A graphical representation of the results is depicted in Figure 6.

\section{Discussion}

Here we show that specific microRNAs are differentially regulated between the main tumor cells of cases with and without epithelial-mesenchymaltransition-like tumor budding and between main tumor and tumor budding cells of cases with tumor budding. An inverse correlation in the expression of target genes involved in epithelial-mesenchymal transition was observed for some of these microRNAs, suggesting that they are directly implicated in the induction of the prognostically unfavorable tumor budding phenotype.

Among the most interesting microRNAs in this regard are the miR-200 family members, miR-200b, miR-200c, and miR-205. This functionally related microRNA family has been shown to play a crucial role in cancer through its strong suppressive effects on cell transformation, proliferation, migration, invasion, tumor growth, and metastasis. ${ }^{29-31}$ Especially, overexpression of miR-200c has been shown to inhibit chemoresistance, invasion, and colony formation of human pancreatic cancer stem cells in pancreatic cancer cell lines. ${ }^{32}$ In accordance to this, we find a marked downregulation of miR-200c and miR-205 in all tumor cells in cases with tumor budding, suggesting that loss of miR-200c and miR-205 expression and abrogation of their suppressive role on migration, taking place already in the main tumor, is a critical step for the formation of tumor buds. Moreover, in cases with tumor budding, miR-200b was significantly reduced in tumor buds compared to main tumor, suggesting that
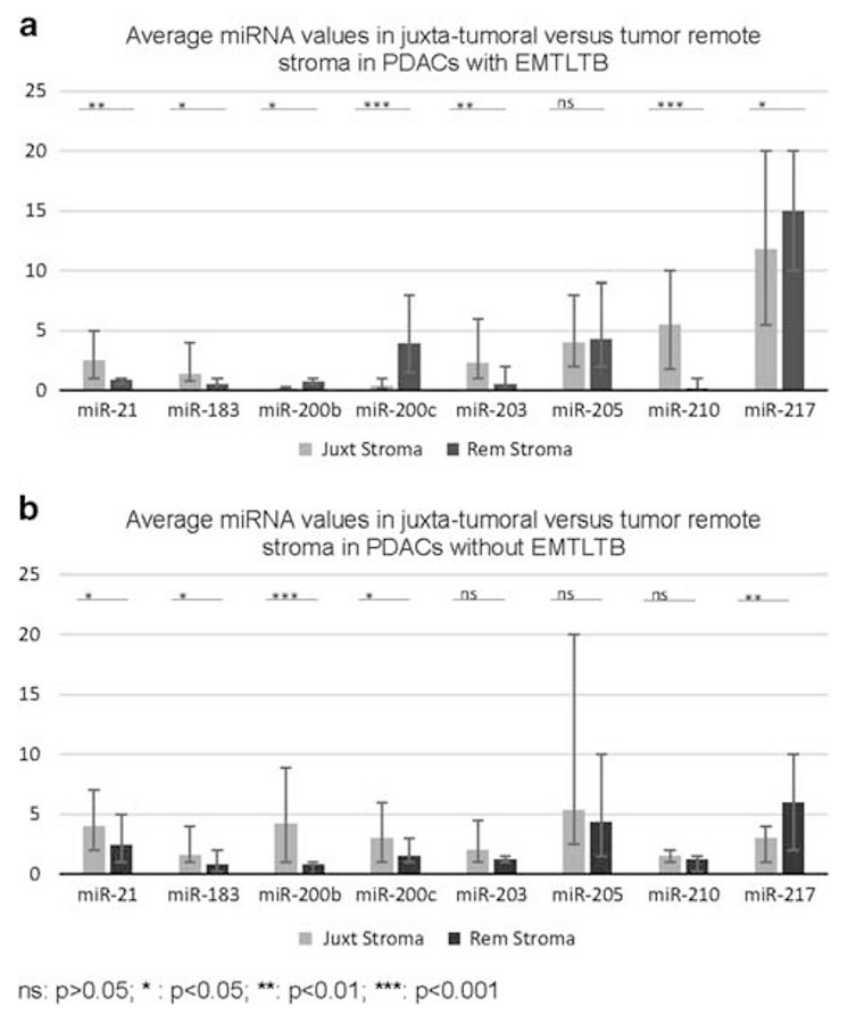

Figure 4 Expression of microRNAs in the juxta-tumoral stromal cells, in pancreatic ductal adenocarcinomas with (a) and without (b) tumor budding, in comparison with tumor-remote stroma.

functionally related microRNAs act in a concerted action to induce tumor bud formation. Interestingly, the expression of miR-200b and miR-200c correlated negatively with the expression of ZEB1 and ZEB2 in the tumor microenvironment of pancreatic cancer. A negative feedback loop between ZEB proteins and microRNA-200 family has been described in various carcinoma models ${ }^{33-35}$ and is considered to constitute the molecular basis for stabilization of either the epithelial or the mesenchymal state in the context of epithelial-mesenchymal transition. ${ }^{36}$ Our findings suggest that this mechanism is also important for tumor bud formation in pancreatic cancer.

On the basis of bioinformatics target prediction (http://www.targetscan.org), miR-203 is able to bind to ZEB1 and ZEB2, but experimental evidence is still missing. In the present study, miR-203 and miR-200b give rise to a similar expression pattern in pancreatic ductal adenocarcinomas with or without tumor budding. Consistent with this finding, both microRNAs are predicted to bind to the same target genes. In the literature, there are contradicting results concerning miR-203 with some studies supporting a tumor promoting and others a tumor suppressive role. ${ }^{37,38}$ However, it has also been suggested that miR-203 has a suppressing role on epithelialmesenchymal transition, by interfering with the TGFb-SMAD3 pathway. ${ }^{39}$ In our study, miR-203 expression showed no consistent correlation with 

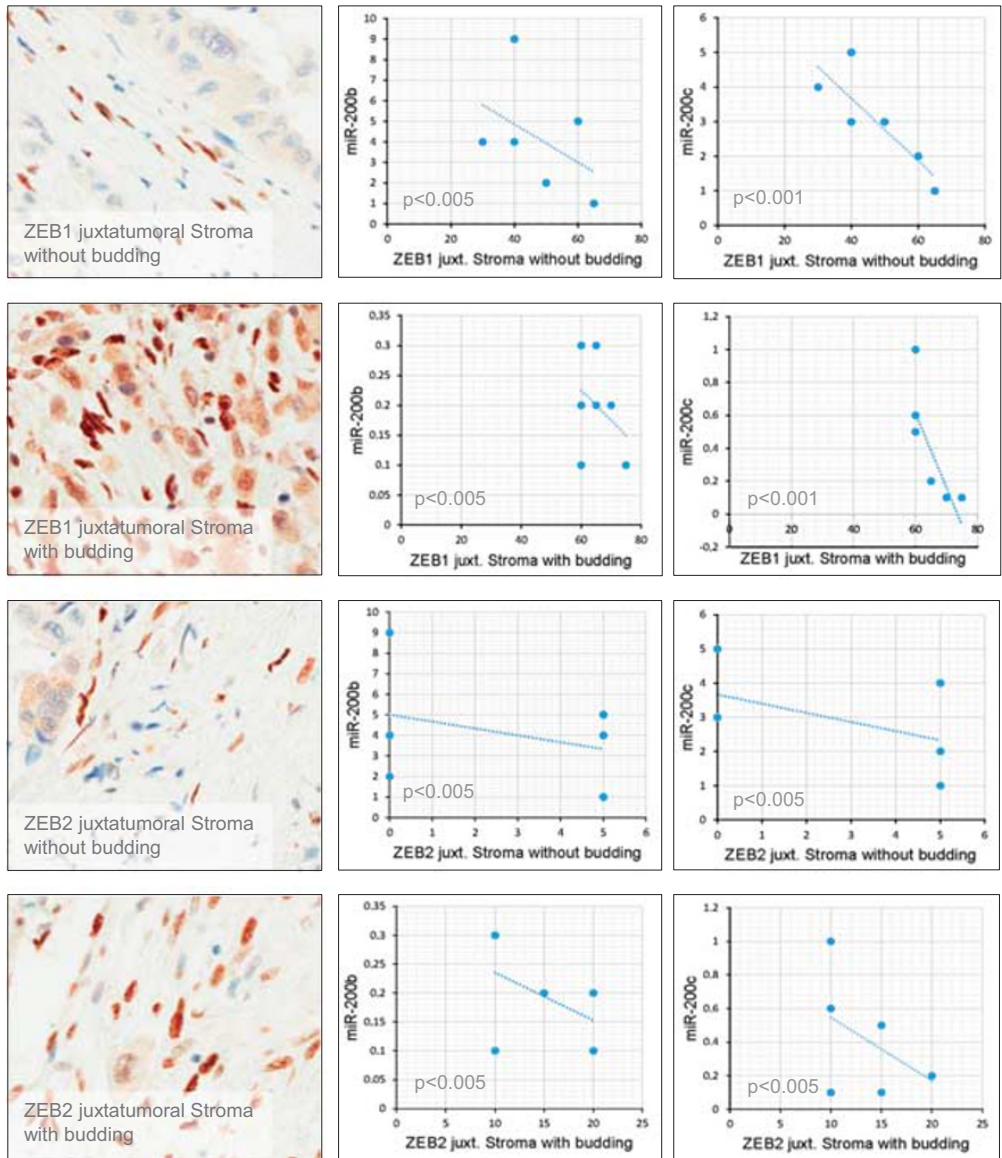
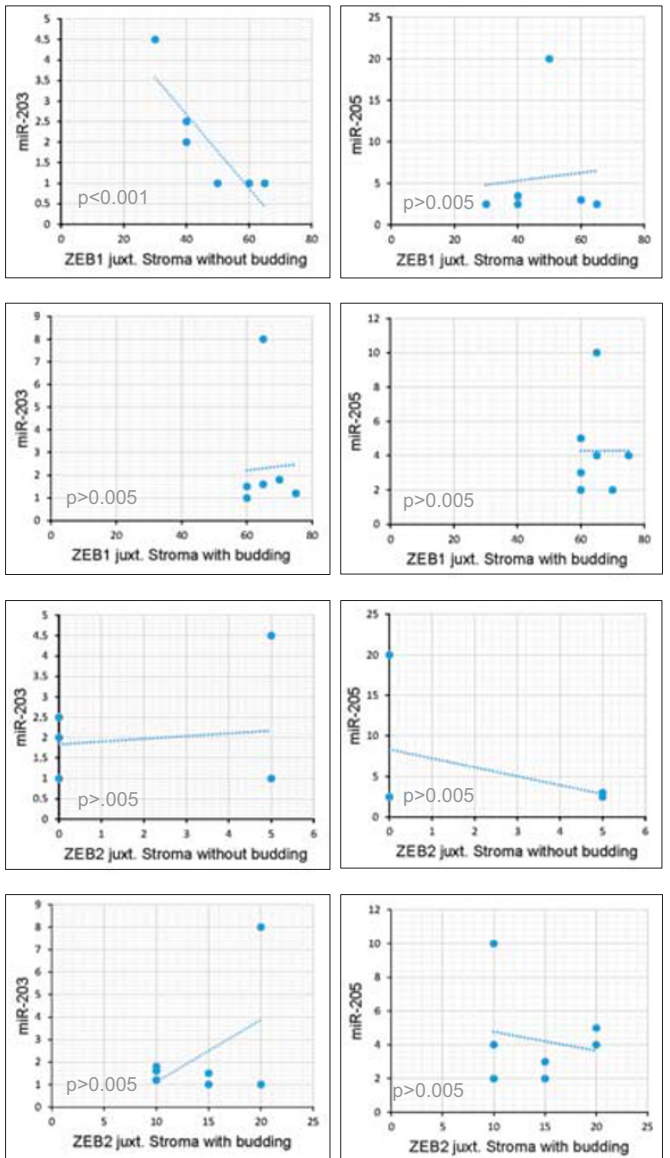

Figure 5 Correlation between ZEB1 and microRNA expression in the juxta-tumoral stromal cells of cases without (first row) and with tumor budding (second row), as well as between ZEB2 and microRNA expression in the juxta-tumoral stromal cells of cases without (third row) and with tumor budding (fourth row), concerning the microRNAs miR-200b, miR-200c, miR-203, and miR-205.

ZEB1 or ZEB2 expression, suggesting that it may affect epithelial-mesenchymal transition independently of ZEB1/ZEB2.

MiR-21 is known to promote epithelial-mesenchymal transition, ${ }^{40-43}$ while miR-183 suppresses apoptosis and promotes proliferation, ${ }^{44-46}$ functions that are necessary for the survival and further growth of the tumor buds. According to this, an aggravated upregulation of miR-21 and miR-183 was seen in the tumor buds compared to main tumor. However, both microRNAs were overexpressed in the tumor cells independently of tumor budding. MiR-217 was found to be downregulated in all tumor cells independently of tumor budding, consistent with its known function as a tumor-suppressing microRNA. ${ }^{47,48}$

In addition, our findings suggest that microRNAs are involved in the crosstalk between tumor and stromal cells, which is increasingly believed to be involved in the neoplastic progression of pancreatic cancer. ${ }^{2-6}$ Indeed, communication between cancer and stromal cells via microvesicles containing microRNAs may represent one way by which tumor cells can modify their microenvironment. This is particularly important for the regulation of further tumor growth at the microenvironment of the invasive front. Aggressive pancreatic cancer cases show at the invasive front high density of dissociative growing cells with epithelial-mesenchymaltransition-like features (tumor buds). Tumor buds have reduced E-cadherin expression and are surrounded by stromal cells overexpressing the E-cadherin repressors ZEB1 and ZEB2. ${ }^{19}$ In these cases, we show that miR-200b and miR-200c are significantly downregulated not only in the tumor buds, but also in the juxta-tumoral stromal cells, this being correlated with the high-level expression of ZEB1 and ZEB2. On the contrary, pancreatic cancer cases with an upregulation of miR-200c and miR-200b in the juxta-tumoral stroma show reduced expression of E-cadherin repressors ZEB1 and ZEB2. These tumors show a retained E-cadherin expression and absence or low density of tumor buds at the invasive front. Our findings in human pancreatic cancer material confirm previous findings from in vitro and mice experiments ${ }^{49,50}$ highly suggesting that the balance between E-cadherin expression by the tumor cells on one hand and the expression of E-cadherin repressors by the stromal cells on the other hand is finely tuned by the expression of miR-200 family in the 


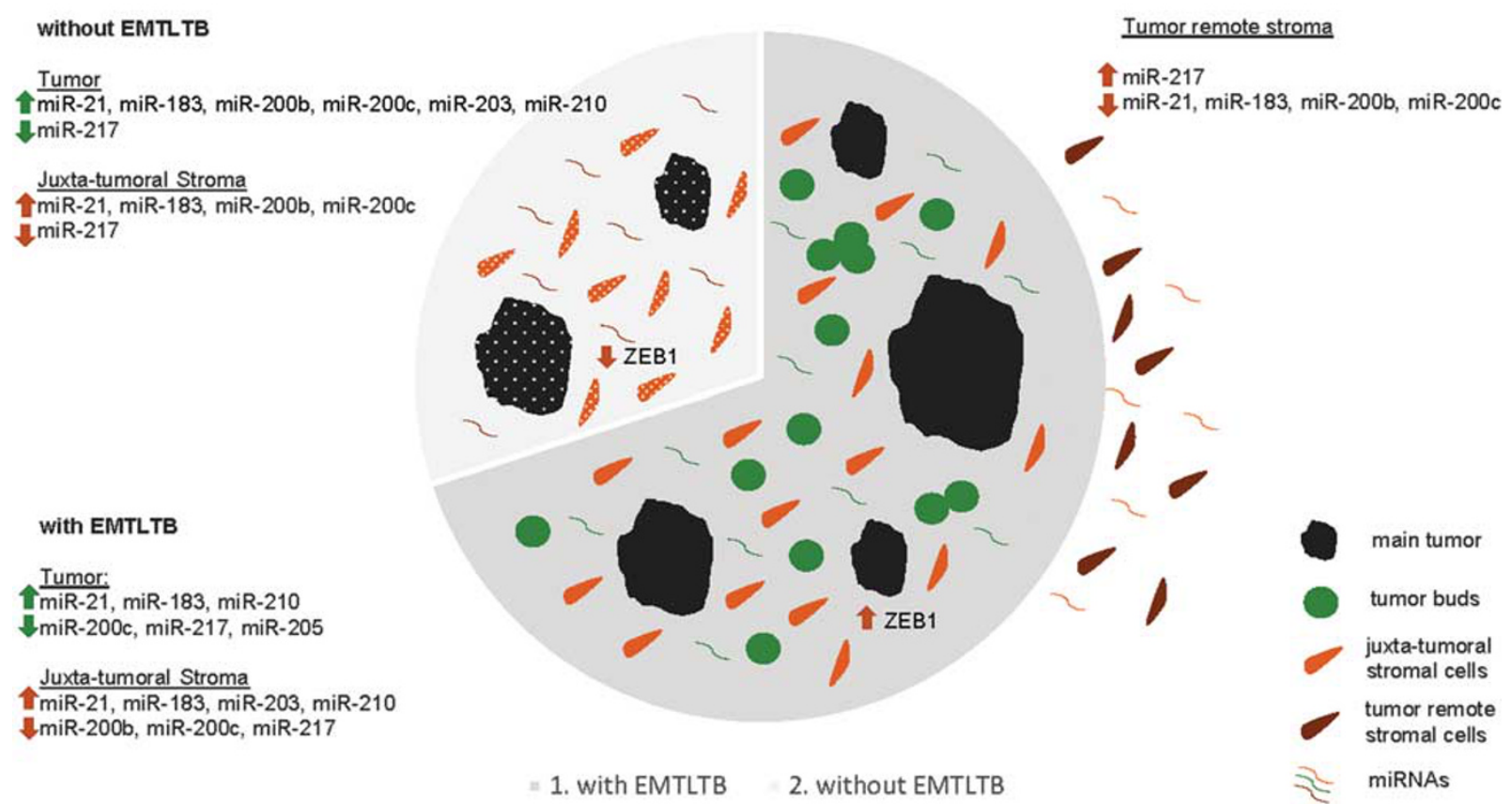

Figure 6 Graphic representation of the differences between pancreatic ductal adenocarcinomas with (a) or without (b) tumor budding.

tumor microenvironment. Moreover, stromal cells seem to regulate the expression of the tumor- and epithelial-mesenchymal-transition-promoting miR-210, exhibiting an upregulation in cases with and a downregulation in cases without tumor budding. Our results are consistent with a previous study showing that stellate cells induced miR-210 upregulation in co-culture experiments with Panc-1 cells, while inhibition of miR-210 expression decreased migration and expression of SNAIL-1, and increased the membrane-associated expression of $\beta$-catenin in cancer cells. ${ }^{51}$ All these findings imply that stromal cells actively influence the differential microRNA expression within the tumor microenvironment in pancreatic cancer.

Furthermore, miR-21 and miR-183 were upregulated and miR-217 was downregulated in the juxtatumoral stromal cells in all cases, analogous to their expression profile in the tumor cells. We could recently show that increased levels of miR-21 in stromal cells were associated with the loss of it's target protein PTEN, especially at the microenvironment of the invasive front of pancreatic ductal adenocarcinoma, which correlated with increased metastatic potential. ${ }^{52}$ MiR-205 was not dysregulated in the stromal cells in agreement with recently published work depicting miR-205 as a purely epithelial microRNA in pancreatic cancer. ${ }^{53}$

This study has several major findings: analysis is based on a well-characterized cohort of pancreatic cancer cases and is designed in a hypothesis-driven approach employing advanced techniques for accurate examination of microRNA levels, while
microRNA analysis is performed on human pancreatic cancer tissue reflecting the genuine state of the tumor microenvironment. Moreover, the expression of microRNAs was examined on material from several areas of importance, including non-neoplastic populations, main tumor, tumor buds, and stromal cells, which were separately analyzed to achieve a comprehensive picture of the microRNA levels in the tumoral and non-tumoral microenvironment.

In conclusion, our findings provide evidence for the dysregulation of specific microRNAs in the tumor microenvironment of pancreatic ductal adenocarcinoma, which may have an impact on the tumor budding phenotype. Since tumor budding is a powerful prognostic factor for pancreatic cancer, microRNAs regulating tumor bud formation can be regarded as attractive therapeutic targets. Moreover, by emphasizing the role of the stromal cells in tumor progression, our results support the concept of novel approaches of therapeutic intervention focusing on the interactions between cancer and stromal cells.

\section{Acknowledgments}

This project was supported by the Bernese Cancer League, the Werner and Hedy Berger-Janser Foundation, and the Foundation for Clinical-Experimental Tumor-Research. The funders had no involvement in the study design; in the collection, analysis, and interpretation of the data; in the writing of the report; and in the decision to submit the paper for publication. 


\section{Disclosure/conflict of interest}

The authors declare no conflict of interest.

\section{References}

1 Hidalgo M, Cascinu S, Kleeff J, et al. Addressing the challenges of pancreatic cancer: future directions for improving outcomes. Pancreatology 2015;15:8-18.

2 Apte MV, Wilson JS, Lugea A, et al. A starring role for stellate cells in the pancreatic cancer microenvironment. Gastroenterology 2013;144:1210-1219.

3 Yeung TL, Leung CS, Li F, et al. Targeting stromalcancer cell crosstalk networks in ovarian cancer treatment. Biomolecules 2016;6:3.

4 Haqq J, Howells LM, Garcea G, et al. Pancreatic stellate cells and pancreas cancer: current perspectives and future strategies. Eur J Cancer 2014;50:2570-2582.

5 Zhou B, Chen WL, Wang YY, et al. A role for cancerassociated fibroblasts in inducing the epithelial-tomesenchymal transition in human tongue squamous cell carcinoma. J Oral Pathol Med 2014;43:585-592.

6 Chu TY, Yang JT, Huang TH, et al. Crosstalk with cancer-associated fibroblasts increases the growth and radiation survival of cervical cancer cells. Radiat Res 2014;181:540-547.

7 Tanaka M, Hashiguchi Y, Ueno H, et al. Tumor budding at the invasive margin can predict patients at high risk of recurrence after curative surgery for stage II, T3 colon cancer. Dis Colon Rectum 2003;46:1054-1059.

8 Lugli A, Karamitopoulou E, Zlobec I. Tumour budding: a promising parameter in colorectal cancer. Br J Cancer 2012;106:1713-1717.

9 Karamitopoulou E, Zlobec I, Born D, et al. Tumour budding is a strong and independent prognostic factor in pancreatic cancer. Eur J Cancer 2013;49:1032-1039.

10 O'Connor K, Li-Chang HH, Kalloger SE, et al. Tumor budding is an independent adverse prognostic factor in pancreatic ductal adenocarcinoma. Am J Surg Pathol 2015;4:472-478.

11 Olson P, Lu J, Zhang H, et al. MicroRNA dynamics in the stages of tumorigenesis correlate with hallmark capabilities of cancer. Genes 2009;23:2152-2165.

12 Frampton AE, Castellano L, Colombo T, et al. MicroRNAs cooperatively inhibit a network of tumor suppressor genes to promote pancreatic tumor growth and progression. Gastroenterology 2014;146:268-277.

13 Stahlhut Espinosa CE, Slack FJ. The role of microRNAs in cancer. Yale J Biol Med 2006;79:131-140.

14 Stahlhut C, Slack FJ. MicroRNAs and the cancer phenotype: profiling, signatures and clinical implications. Genome Med 2013;5:111.

15 Tessitore A, Cicciarelli G, Mastroiaco V, et al. Therapeutic use of microRNAs in cancer. Anticancer Agents Med Chem 2015;16:7-19.

16 Fellenberg J, Sähr H, Kunz P, et al. Restoration of miR-127-3p and miR-376a-3p counteracts the neoplastic phenotype of giant cell tumor of bone derived stromal cells by targeting COA1, GLE1 and PDIA6. Cancer Lett 2016;371:134-141.

17 Passadouro M, Faneca H. Managing pancreatic adenocarcinoma: a special focus in microRNA gene therapy. Int J Mol Sci 2016;17:718.

18 Kakar S, Pawlik TM, Allen PJ, et al. Exocrine pancreas. Pancreatic adenocarcinoma. In: Amin MB (ed). AJCC
Cancer Staging Manual, 8th edn. Springer-Verlag: New York, 2016.

19 Galván JA, Zlobec I, Wartenberg M, et al. Expression of E-cadherin repressors SNAIL, ZEB1 and ZEB2 by tumour and stromal cells influences tumour-budding phenotype and suggests heterogeneity of stromal cells in pancreatic cancer. Br J Cancer 2015;112: 1944-1950.

20 Bandi N, Vassella E. MiR-34a and miR-15a/16 are coregulated in non-small cell lung cancer and control cell cycle progression in a synergistic and Rb-dependent ?thyc?> manner. Mol Cancer 2011;10:55.

21 McShane LM, Altman DG, Sauerbrei W, et al. Statistics subcommittee of the NCI-EORTC Working Group on cancer diagnostics. Reporting recommendations for tumour MARKer prognostic studies (REMARK). Br J Cancer 2005;93:387-391.

22 Nishijima N, Seike M, Soeno C, et al. MiR-200/ZEB axis regulates sensitivity to nintedanib in non-small cell lung cancer cells. Int J Oncol 2016;48:937-944.

$23 \mathrm{Li} \mathrm{Y,} \mathrm{Zeng} \mathrm{C,} \mathrm{Tu} \mathrm{M,} \mathrm{et} \mathrm{al.} \mathrm{MicroRNA-200b} \mathrm{acts} \mathrm{as} \mathrm{a}$ tumor suppressor in osteosarcoma via targeting ZEB1. Onco Targets Ther 2016;9:3101-3111.

24 Damiano V, Brisotto G, Borgna S, et al. Epigenetic silencing of miR-200c in breast cancer is associated with aggressiveness and is modulated by ZEB1. Genes Chromosomes Cancer 2017;56:147-158.

25 Eggers JC, Martino V, Reinbold R, et al. MicroRNA miR-200b affects proliferation, invasiveness and stemness of endometriotic cells by targeting ZEB1, ZEB2 and KLF4. Reprod Biomed Online 2016;32:434-445.

26 Jiao A, Sui M, Zhang L, et al. MicroRNA-200c inhibits the metastasis of non-small cell lung cancer cells by targeting ZEB2, an epithelial-mesenchymal transition regulator. Mol Med Rep 2016;13:3349-3355.

27 Lee JY, Park MK, Park JH, et al. Loss of the polycomb protein Mel-18 enhances the epithelial-mesenchymal transition by ZEB1 and ZEB2 expression through the downregulation of miR-205 in breast cancer. Oncogene 2014;33:1325-1335.

28 Wellner U, Schubert J, Burk UC, et al. The EMTactivator ZEB1 promotes tumorigenicity by repressing stemness-inhibiting microRNAs. Nat Cell Biol 2009;11: 1487-1495.

29 Humphries B, Yang C. The microRNA-200 family: small molecules with novel roles in cancer development, progression and therapy. Oncotarget 2015;6: 6472-6498.

$30 \mathrm{Xu} \mathrm{C}, \mathrm{Li} \mathrm{M}$, Zhang L, et al. MicroRNA-205 suppresses the invasion and epithelial-mesenchymal transition of human gastric cancer cells. Mol Med Rep 2016;13: 4767-4773.

31 Zhang C, Long F, Wan J, et al. MicroRNA-205 acts as a tumor suppressor in osteosarcoma via targeting RUNX2. Oncol Rep 2016;35:3275-3284.

$32 \mathrm{Ma}$ C, Huang T, Ding YC, et al. MicroRNA-200c overexpression inhibits chemoresistance, invasion and colony formation of human pancreatic cancer stem cells. Int J Clin Exp Pathol 2015;8:6533-6539.

33 Burk U, Schubert J, Wellner U, et al. A reciprocal repression between zeb1 and members of the mir-200 family promotes EMT and invasion in cancer cells. EMBO Rep 2008;9:582-589.

34 Bracken CP, Gregory PA, Kolesnikoff N, et al. A doublenegative feedback loop between zeb1-sip1 and the microRNA-200 family regulates epithelial-mesenchymal transition. Cancer Res 2008;68:7846-7854. 
35 Gregory PA, Bert AG, Paterson EL, et al. The mir-200 family and mir-205 regulate epithelial to mesenchymal transition by targeting zeb1 and sip1. Nat Cell Biol 2008;10:593-601.

36 Park SM, Gaur AB, Lengyel E, et al. The mir-200 family determines the epithelial phenotype of cancer cells by targeting the e-cadherin repressors zeb1 and zeb2. Genes Dev 2008;22:894-907.

37 Zhao G, Guo Y, Chen Z, et al. MiR-203 functions as a tumor suppressor by inhibiting epithelial to mesenchymal transition in ovarian cancer. J Cancer Sci Ther 2015;7:34-43.

38 Xiaohong Z, Lichun F, Na X, et al. MiR-203 promotes the growth and migration of ovarian cancer cells by enhancing glycolytic pathway. Tumour Biol 2016;37: 14989-14997.

$39 \mathrm{Hu}$ H, Xu Z, Li C, et al. MiR-145 and miR-203 represses TGF- $\beta$-induced epithelial-mesenchymal transition and invasion by inhibiting SMAD3 in non-small cell lung cancer cells. Lung Cancer 2016;97:87-94.

40 Shi J. Considering exosomal miR-21 as a biomarker for cancer. J Clin Med 2016;5:42.

41 Kadera BE, Li L, Toste PA, et al. MicroRNA-21 in pancreatic ductal adenocarcinoma tumor-associated fibroblasts promotes metastasis. PLoS ONE 2013;8: e71978.

42 Donahue TR, Nguyen AH, Moughan J, et al. Stromal MicroRNA-21 levels predict response to 5-fluorouracil in patients with pancreatic cancer. J Surg Oncol 2014;110:952-959.

43 Liu Z, Wang J, Guo C, et al. MicroRNA-21 mediates epithelial-mesenchymal transition of human hepatocytes via PTEN/Akt pathway. Biomed Pharmacother 2015;69: 24-28.

44 Ma Y, Liang AJ, Fan YP, et al. Dysregulation and functional roles of miR-183-96-182 cluster in cancer cell proliferation, invasion and metastasis. Oncotarget 2016;7:42805-42825.
45 Miao F, Zhu J, Chen Y, et al. MicroRNA-183-5p promotes the proliferation, invasion and metastasis of human pancreatic adenocarcinoma cells. Oncol Lett 2016;11:134-140.

46 Yang M, Liu R, Li X, et al. MiRNA-183 suppresses apoptosis and promotes proliferation in esophageal cancer by targeting PDCD4. Mol Cells 2014;37:873-880.

47 Deng S, Zhu S, Wang B, et al. Chronic pancreatitis and pancreatic cancer demonstrate active epithelialmesenchymal transition profile, regulated by miR-217SIRT1 pathway. Cancer Lett 2014;355:184-191.

48 Wang H, Dong X, Gu X, et al. The microRNA-217 functions as a potential tumor suppressor in gastric cancer by targeting GPC5. PLoS ONE 2015;10:e0125474.

49 Kurashige J, Mima K, Sawada G, et al. Epigenetic modulation and repression of miR-200b by cancerassociated fibroblasts contribute to cancer invasion and peritoneal dissemination in gastric cancer. Carcinogenesis 2015;36:133-141.

50 Perdigão-Henriques R, Petrocca F, Altschuler G, et al. MiR-200 promotes the mesenchymal to epithelial transition by suppressing multiple members of the Zeb2 and Snail1 transcriptional repressor complexes. Oncogene 2016;35:158-172.

51 Takikawa T, Masamune A, Hamada S, et al. MiR-210 regulates the interaction between pancreatic cancer cells and stellate cells. Biochem Biophys Res Commun 2013;437:433-439.

52 Wartenberg M, Centeno I, Haemmig S, et al. PTEN alterations of the stromal cells characterize an aggressive subpopulation of pancreatic cancer with enhanced metastatic potential. Eur J Cancer 2016;65:80-90.

53 Han S, Gonzalo DH, Feely M, et al. The pancreatic tumor microenvironment drives changes in miRNA expression that promote cytokine production and inhibit migration by the tumor associated stroma. Oncotarget; e-pub ahead of print 20 July 2016; doi: 10.18632/oncotarget.10722.

Supplementary Information accompanies the paper on Modern Pathology website (http://www.nature.com/ modpathol) 